\title{
Design of a Fault-Injectable Fleischer-Laker Switched-Capacitor Biquad for verifying the Static Linear Behavior Fault Model
}

\author{
Long-Yi Lin and Hao-Chiao Hong \\ Department of Electrical and Computer Engineering, \\ National Chiao Tung University, Hsinchu, Taiwan, R.O.C. \\ E-mail: d6943e@yahoo.com.tw, hchong@cn.nctu.edu.tw
}

\begin{abstract}
This paper presents the design of a fully-differential low-pass SC biquad filter that allows injecting the parametric and catastrophic faults to it. The design is for the verification of the static linear behavior (SLB) fault model which covers multiple parametric and catastrophic analog faults of sampleddata systems. The SLB fault model assumes that a circuit under test (CUT) has a fixed z-domain transfer function (TF) template and the faults alter nothing but the coefficients of the TF. To verify this basic assumption, we first retrieve all coefficients of the TF of the fault-injected biquad from the test results of a three-tone test following the diagnosis-after-test (DAT) procedure. Then, we conduct simulations with other stimulus tones and check if the tested responses fit in those predicted by the retrieved TF. Experimental results verify that the fixed TF template assumption holds for all the faults that we injected.
\end{abstract}

\section{INTRODUCTION}

A practical fault model is essential for designing efficient tests to reduce the test cost and to ensure the test quality. The stuck-at fault model is the most successful example. It is the most popular fault model for digital circuits and enables modern VLSI designs. Without the stuck-at fault model, the test cost and thus the product cost would be too high to be acceptable.

However, the fault model for analog circuits is not as popular as that for digital circuits [1]. Too long fault simulation time and unverified fault assumptions are two issues that make analog fault models not widely accepted [2], [3]. Consequently, functional tests become the standard tests for analog circuits in industry. The major issue of adopting functional tests is engineers don't know how many and what stimuli are sufficient to ensure the test quality due to the lack of an adequate analog fault model.

Switched-capacitor (SC) circuits are very popular for designing the analog circuits in modern mixed-signal IC due to their accurate frequency responses, good linearity, and wide dynamic ranges [4], [5]. Therefore, how to test SC circuits efficiently has gained much attentions [6]-[9]. Reference [10] proposed a static linear behavior (SLB) analog fault model for linear and time-invariant (LTI) sampled-data analog circuits including the SC circuits. The SLB fault model assumes the transient responses of the circuit under test (CUT) are fully settled within every clock cycle and thus the z-domain transfer function (TF) of the CUT has a fixed template but may with different coefficients no matter what faults the CUT has. Different from conventional analog fault models that rely on the fault simulation results to make the pass/fail decisions [11]-[13], the SLB fault model decides a faulty CUT by comparing the design specification with the frequency responses of the tested $\mathrm{z}$-domain TF whose coefficients are derived from the test results.

The fault list of the SLB fault model covers not only parametric faults such as the open loop gains of the operational amplifiers (OPAMPs) and the capacitor ratios of the capacitors, but also catastrophic faults of the switches, OPAMPs, and capacitors of the CUT. In particular, the SLB analog fault model deals with multiple catastrophic faults and parametric faults that resulted in too long fault simulation time with conventional analog fault models.

A diagnosis-after-test (DAT) procedure that can test for multiple faults of the CUT has been proposed in [10]. Reference [14] took a versatile Fleischer-Laker SC biquad as an example CUT to demonstrate the DAT procedure. Behavioral simulations were conducted to show how to use the threetone test results to estimate all the coefficients of the CUT's TFs. The resulted coefficients are then used to find all of the capacitor ratios on the CUT so as to achieve a $100 \%$ parametric fault coverage defined by the SLB analog fault model.

In this paper, we design a second-order low-pass filter with the E-circuit topology as the CUT to verify the basic assumption of the SLB analog fault model: The z-domain TF of the CUT has a fixed template no matter what faults the CUT has. Several example parametric and catastrophic faults are designed to be injected into the CUT. After every fault injection, a three-tone test is conducted to estimate the coefficients of the faulty CUT's TF following the DAT procedure. Then, additional tests with different test tones are conducted. Finally, we compare the test results with the predictions of the estimated TF. If they fit in to each other in all cases, it justifies the correctness of the SLB fault model.

This paper is organized as follows. Section II depicts the circuit design and shows how to inject the faults to the SC biquad. Experimental results are given in Section III. Finally, 
Table I

DESIGN SPECIFICATION OF THE LOW-PASS FILTER.

\begin{tabular}{c|c|c|c|c}
\hline Specification & Min & Typical & Max & Unit \\
\hline Sample frequency (fs) & & 2500 & & $\mathrm{kHz}$ \\
\hline Passband (fpass) & & 20 & & $\mathrm{kHz}$ \\
\hline Passband gain & & 0 & & $\mathrm{~dB}$ \\
\hline Passband ripple (Amin) & -1.2 & & 1.2 & $\mathrm{~dB}$ \\
\hline Stopband (fstop) & & 400 & & $\mathrm{kHz}$ \\
\hline Stopband attenuation (Amax) & 30 & & & $\mathrm{~dB}$ \\
\hline
\end{tabular}

we draw our conclusions in Section IV.

\section{Design OF the FAult InJECTABle Fleischer-LAKeR SWITCHED-CAPACITOR BIQUAD}

Fig. 1 depicts the schematic of the proposed fault-injectable Fleischer-Laker SC E-circuit biquad. Like most analog circuit designs for IC, the CUT design is fully differential for better noise immunity and common-mode interference rejection. The design was done based on a $0.18 \mu \mathrm{m}$ CMOS process provided by the Chip Implementation Center (CIC), Taiwan. We implemented the OPAMPs with conventional folded cascode topology [15]. Simulation results show the OPAMP design achieves a $71.5 \mathrm{~dB}$ open-loop gain, a $175 \mathrm{MHz}$ unit-gain frequency, and a $73^{\circ}$ phase margin with $4 \mathrm{pF}$ loads.

\section{A. Design of the Circuits under Test}

The Fleischer-Laker SC biquad is used to realize a Butterworth low-pass filter whose passband is $20 \mathrm{kHz}$ with a sampling frequency of $2.5 \mathrm{MHz}$. Table I summarizes the design specification of this filter.

Detailed circuit analysis show the nominal TF of the CUT is

$$
\begin{gathered}
T_{2}(z) \equiv \frac{V_{o 2}(z)}{V_{i}(z)} \\
=-\frac{a_{3}+a_{4} z^{-1}+a_{5} z^{-2}}{1+a_{1} z^{-1}+a_{2} z^{-2}} \\
=-\frac{\frac{C_{I}}{C_{B}}-\left(\frac{C_{I}}{C_{B}}+\frac{C_{J}}{C_{B}}-\frac{C_{A}}{C_{B}} \frac{C_{G}}{C_{D}}\right) z^{-1}+\left(\frac{C_{J}}{C_{B}}-\frac{C_{A}}{C_{B}} \frac{C_{H}}{C_{D}}\right) z^{-2}}{1-\left(2-\frac{C_{A}}{C_{B}} \frac{C_{C}}{C_{D}}-\frac{C_{A}}{C_{B}} \frac{C_{E}}{C_{D}}\right) z^{-1}+\left(1-\frac{C_{A}}{C_{B}} \frac{C_{E}}{C_{D}}\right) z^{-2}}
\end{gathered}
$$

Equation (1) depicts that the coefficients of the CUT's TF are mainly determined by the capacitance values of the nine capacitors $C_{A}, C_{B}, C_{C}, C_{D}, C_{E}, C_{G}, C_{H}, C_{I}$, and $C_{J}$ of the biquad. More precisely, they are determined by the seven capacitor ratios. Following typical filter design procedure, the final designated capacitor ratios of the capacitors are listed in Table II. The frequency responses of the nominal TF of the CUT are plotted in Fig. 2.

\section{B. Fault Injection}

The SLB analog fault model covers both parametric and catastrophic faults. Parametric faults are the results of parameter deviations of components due to process, voltage, and temperature variations. Capacitance values deviations are typical parametric faults in SC circuits. Hence, we add two differential capacitor pairs $C_{A p f}$ and $C_{G p f}$ to the biquad and decompose $C_{E}$ into two capacitors $C_{E 1}$ and $C_{E 2}$. By issuing the control signals $A p$ and $G p$, the total capacitance of the
Table II

CAPACITOR RATIOS AND VALUES OF THE CUT.

\begin{tabular}{c|c|c}
\hline Parameters & Designated ratio & absolute value $(\mathrm{pF})$ \\
\hline$C_{A}$ & $0.20289855 C_{B}$ & 1.288 \\
\hline$C_{B}$ & $1 C_{B}$ & 6.348 \\
\hline$C_{C}$ & $0.07246376 C_{D}$ & 0.460 \\
\hline$C_{D}$ & $1 C_{D}$ & 6.348 \\
\hline$C_{E}=C_{E 1}+C_{E 2}$ & $0.81159420 C_{D}$ & 5.152 \\
\hline$C_{G}$ & $0.10144927 C_{D}$ & 0.644 \\
\hline$C_{H}$ & $0.0289855 C_{D}$ & 0.184 \\
\hline$C_{I}$ & $0.00362318 C_{B}$ & 0.023 \\
\hline$C_{J}$ & $0.00724637 C_{B}$ & 0.046 \\
\hline$C_{A p f}$ & $0.4057971 C_{B}$ & 2.576 \\
\hline$C_{E 1}$ & $0.4057971 C_{D}$ & 2.576 \\
\hline$C_{E 2}$ & $0.4057971 C_{D}$ & 2.576 \\
\hline$C_{G p f}$ & $0.20289854 C_{D}$ & 1.288
\end{tabular}

corresponding capacitors are enlarged respectively to model practical parametric faults in a controlled manner. Similarly, activating the control signal $\overline{E p}$ will reduce the effective capacitance of $C_{E}$ by $C_{E 2}$ to model another kind of parametric fault. The capacitance values of these capacitors are listed in Table II.

On the other hand, a catastrophic fault leads to a different circuit topology and netlist. It has been shown that the catastrophic faults of the capacitors and OPAMPs are equivalent to particular parametric faults [10]. They can be detected during the parametric fault tests. As a result, the SLB fault model assumes only the delay-free and delayed SC branches may suffer from catastrophic faults. Reference [10] shows these catastrophic faults change the coefficients but not the templates of the CUT's TFs, too.

To verify the deductions of the SLB fault model about the catastrophic faults, we add three switches including $S G s$, $S H s$, and $S G o$ to the design as shown in Fig. 1. The aspect ratios of the switches $S G s$ and $S H s$ are designed to be much larger than those of the switches $S G n$ and $S H n$ which are in parallel with them. By keeping $S G s$ or $S H s$ turn-on, a short fault is injected to the corresponding switch. The design allows injecting a stuck-open fault to the biquad, too. The switch $S G o$ can be turn off for injecting the stuck-open fault.

\section{EXPERIMENTAL RESULTS}

To verify the basic assumption of the SLB analog fault model: The z-domain TF of the CUT has a fixed template no matter what faults the CUT has, our test plan follows.

1) Injecting a fault to the CUT.

2) Conducting a three-tone test to estimate the coefficients of the faulty CUT's TF following the procedure proposed in [14].

3) Conducting additional tests with checking tones whose frequencies are different from the three tones.

4) Comparing the test results with the predictions of the estimated TF.

5) Repeat step 1 to 4 for all faults.

If the test responses of the checking tones fit in the frequency responses of the estimated TF in all cases, it justifies the basic assumption of the SLB fault model. 


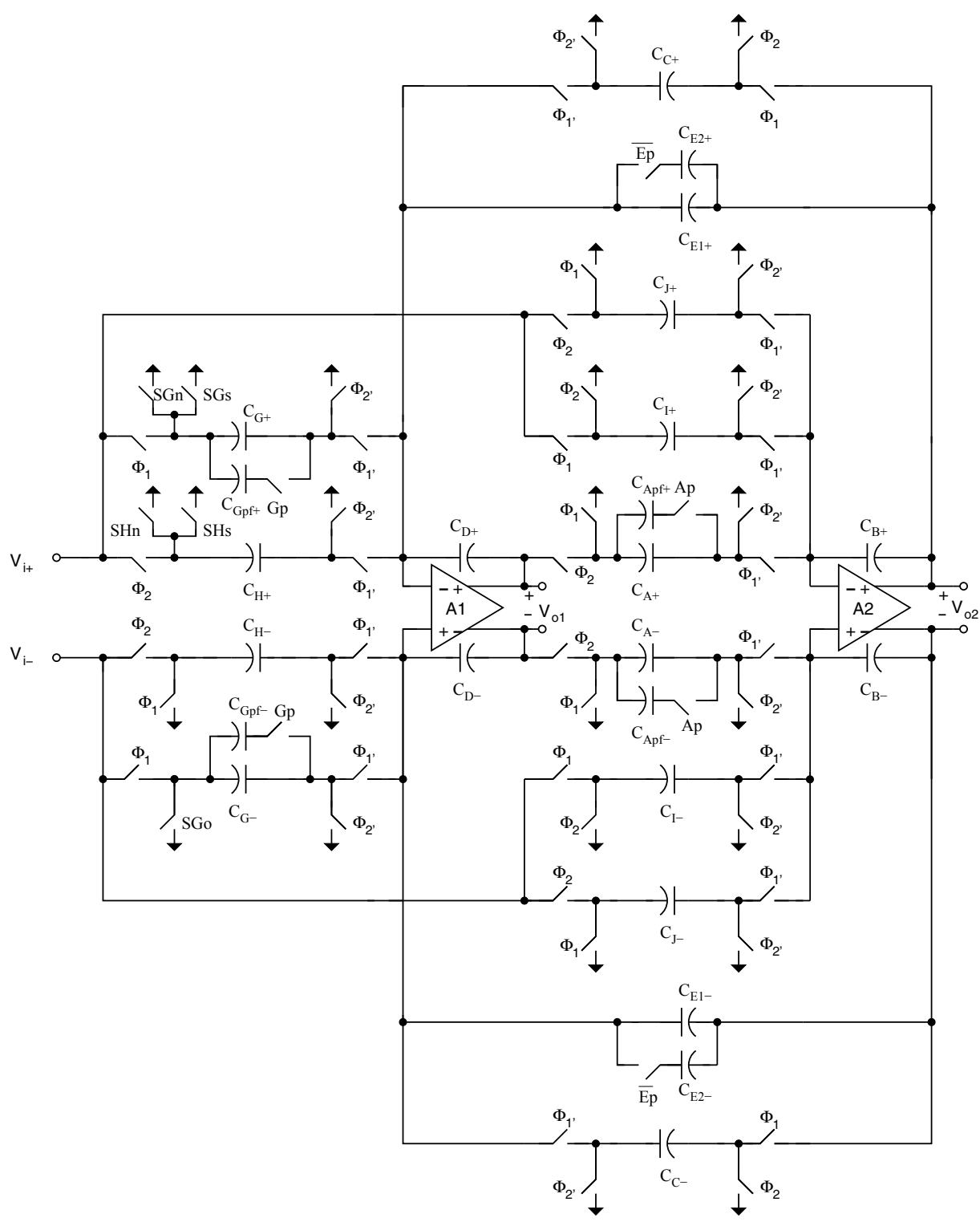

Figure 1. The schematic of the proposed fault-injectable Fleischer-Laker SC biquad.

Reference [10] suggested that a low-frequency tone, a tone around the corner frequency of the CUT, and a high-frequency tone close to the stopband frequency is a good combination for the required three-tone test. This test setup allows the test results to be used not only to compute the coefficients of the CUT's practical TF, but also to check if the CUT conforms to the design specification. Hence, we selected $22 \mathrm{kHz}, 51$ $\mathrm{kHz}$, and $398 \mathrm{kHz}$ as the stimulus tones. In the following, all experimental results are the circuit simulation results of HSPICE.

Fig. 2 shows the simulated output spectra of the CUT without injecting any fault. Following the DAT procedure, we first estimate all the coefficients of the TF, $a_{1}$ to $a_{5}$ of (1), according to the measured data of the three tones. The frequency responses of the resulted TF are plotted as the dashed line in Fig. 3. The white district of Fig. 3 indicates the design window of the CUT. That is, the frequency response plot of the CUT can not cross the gray regions of the figure to pass the test. Otherwise, the CUT fails in the test.

We also plot the targeted frequency responses, the test results of the three tones, and those of the checking tones on the same figure. Interestingly, the frequency responses of the estimated TF are different from the design targets even though all capacitors are assigned to have their nominal values. It is because practical transistors contribute parasitic capacitors and thus alter the coefficients of the TF. Consequently, the CUT's TF slightly differs from the design target. The scenario can be considered as multiple parametric faults occur. Nevertheless, 


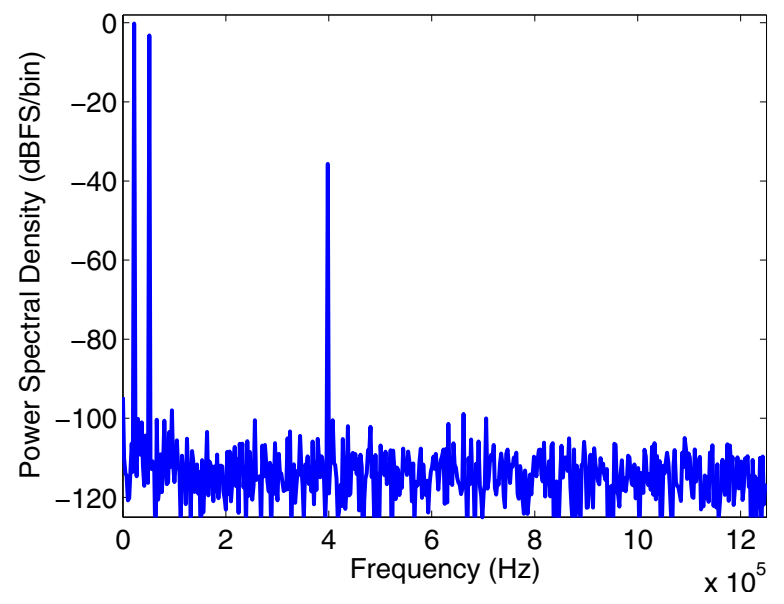

Figure 2. The output spectrum of the CUT with the 3-tone test stimulus.

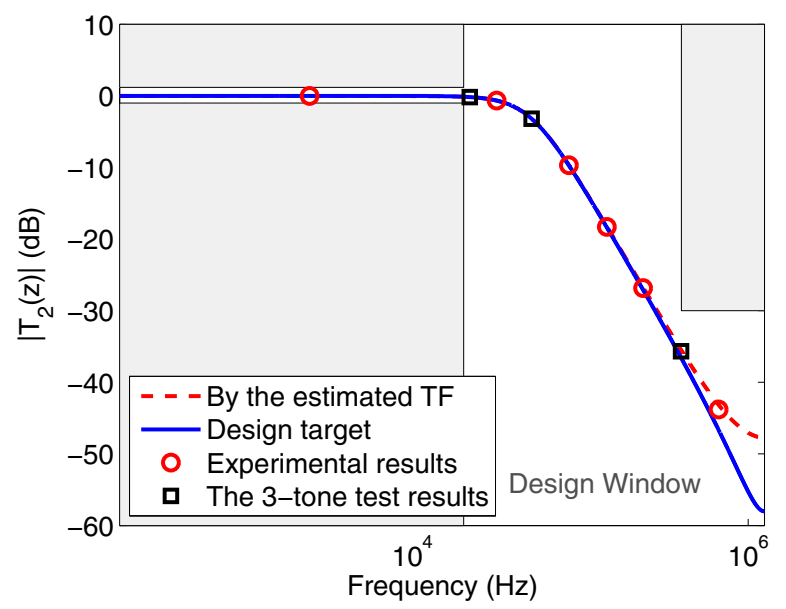

Figure 3. Experimental results of the CUT without fault injection.

the experimental results of the checking stimulus tones fit it the frequency responses of the estimated TF very well.

Fig. 4 shows the simulation results of the CUT when the parametric fault of $C_{G p f}$ is injected. This parametric fault makes the passband gain and the stopband attenuation of the CUT out of the design specification. Even though the CUT fails in the test, the estimated TF still accurately depicts the faulty behavior of the CUT.

Fig. 5 and 6 illustrate the simulation results of the CUT after enabling the parametric fault control signals of $A p$ and $\overline{E p}$, respectively. The enlarged $C_{A}$ leads to a too small minimum stopband attenuation, while the CUT passes the test with the reduced $C_{E}$. Similar to the previous case, the estimated TF well predicts the frequency responses of the CUT in both cases no matter the CUT passes or fails.

Fig. 7 and 8 depicts the experimental results of the CUT when the catastrophic faults are activated. Both catastrophic faults fail the CUT in the test. Yet the estimated TFs success-

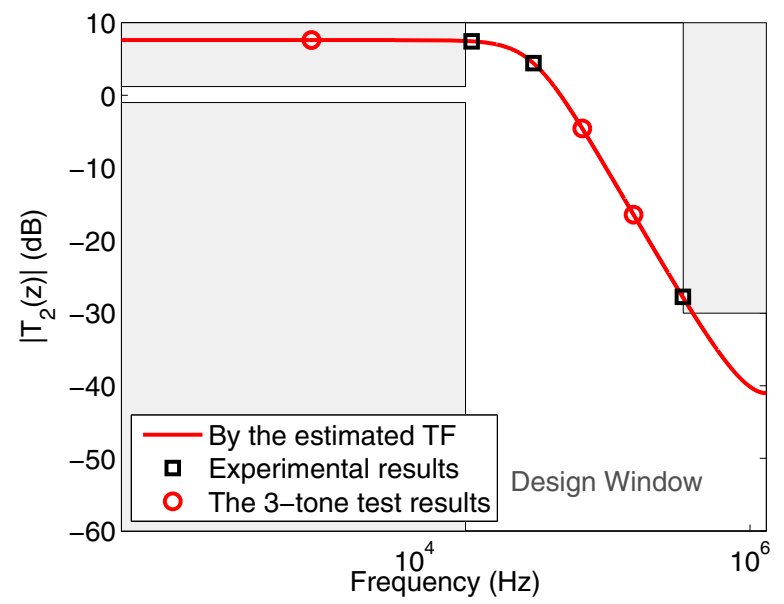

Figure 4. Experimental results of the primary output's magnitude responses after injecting the parametric fault of $C_{G p f}$.

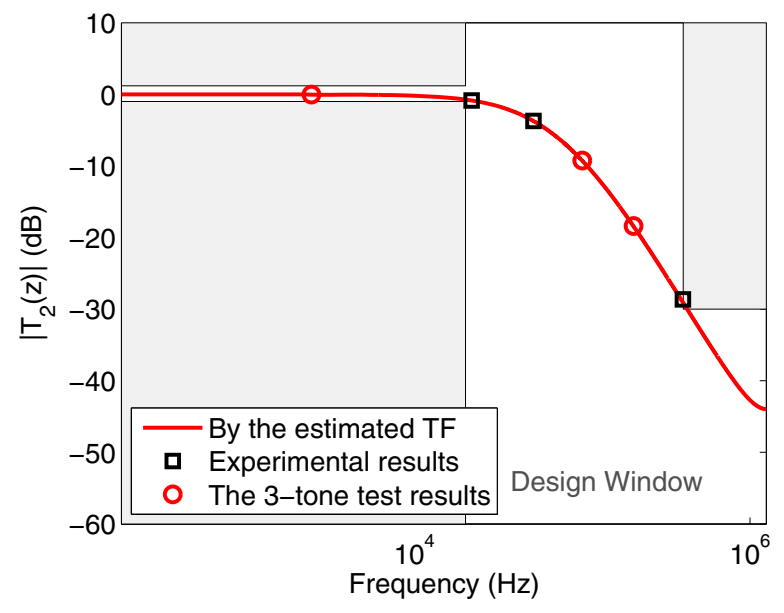

Figure 5. Experimental results with the parametric fault activator $A p$ enabled.

fully depicts the faulty frequency responses of the CUT.

\section{CONCLUSION}

We present the design of a fully-differential low-pass SC biquad filter that allows injecting the parametric and catastrophic faults to it. The design is to verify the basic assumption of the static linear SLB fault model: a CUT has a fixed z-domain TF template and the faults alter nothing but the coefficients of the TF. To verify this assumption, we first retrieve all coefficients of the TF of the fault-injected biquad from the test results of a three-tone test following the diagnosis-after-test (DAT) procedure. Then, we conduct simulations with other stimulus tones and check if the test responses fit in those predicted by the retrieved TF. Experimental results verify that the fixed TF template assumption holds for all the faults that we injected. 


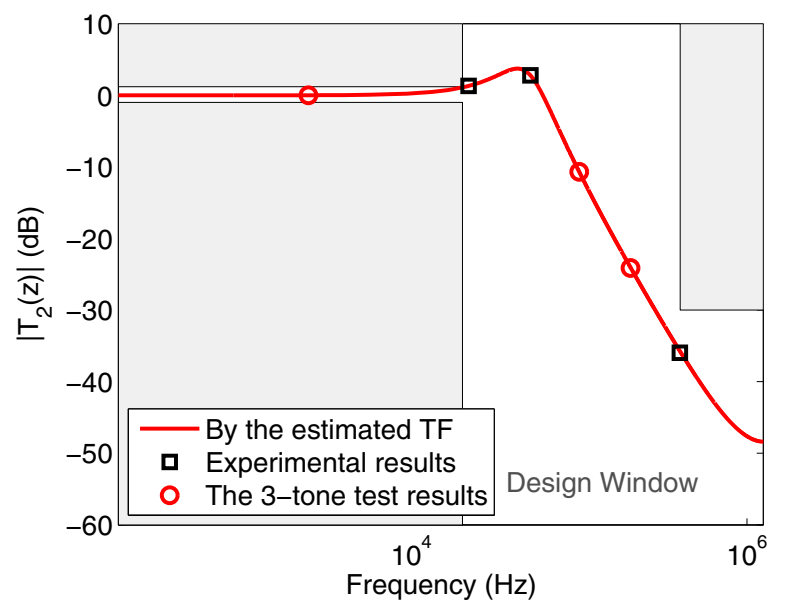

Figure 6. Experimental results with the parametric fault activator Ep enabled.

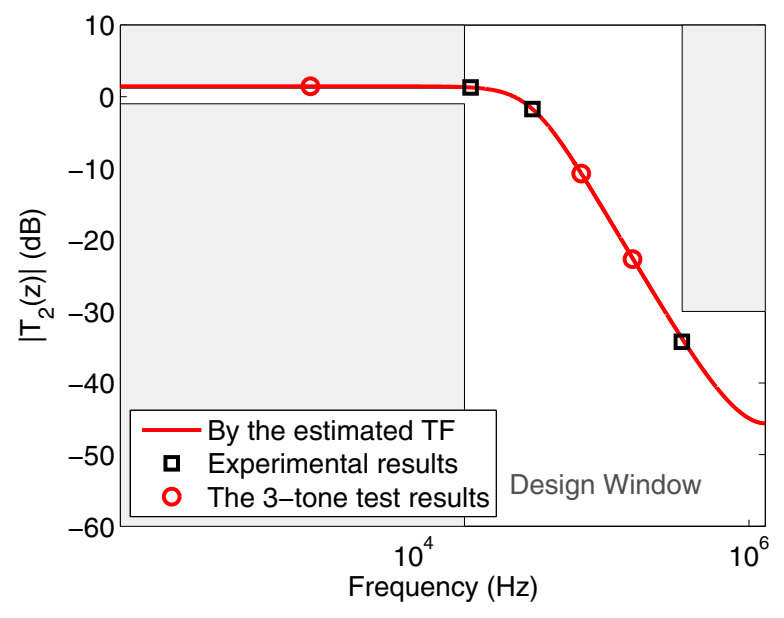

Figure 7. Experimental results when the switch $S H s$ is short.

\section{ACKNOWLEDGMENT}

This work is supported in part by the National Science Council (NSC), Taiwan R.O.C. through the grant NSC 1012221-E-009-166-.

\section{REFERENCES}

[1] L.-T. Wang, C.-W. Wu, and X.-G. Wen, VLSI Test Principles and Architectures. 500 Sansome St., Suite 400, San Francisco, CA 94111: Morgan Kaufmann Publishers, 2006.

[2] M. Soma, "Challenges in analog and mixed-signal fault models," IEEE Circuits and Devices Magazine, vol. 12, no. 1, pp. 16-19, 1996.

[3] C.-Y. Chao, H.-J. Lin, and L. Milor, "Optimal testing of VLSI analog circuits," IEEE Trans. Computer-Aided Design of Integrated Circuits and Systems, vol. 16, no. 1, pp. 58-77, Jan. 1997.

[4] R. Gregorian and G. C. Temes, Analog MOS integrated circuits for signal processing. N.Y.: Wiley-Interscience, 1986.

[5] K. R. Laker and W. M. Sansen, Design of analog integrated circuits and systems. Hightstown, N.J.: McGraw-Hill, 1994.

[6] M. Soma and V. Kolarik, "A design-for-test technique for switchedcapacitor filters," in Proc. IEEE VLSI Test Symp. (VTS), 1994, pp. 4247.

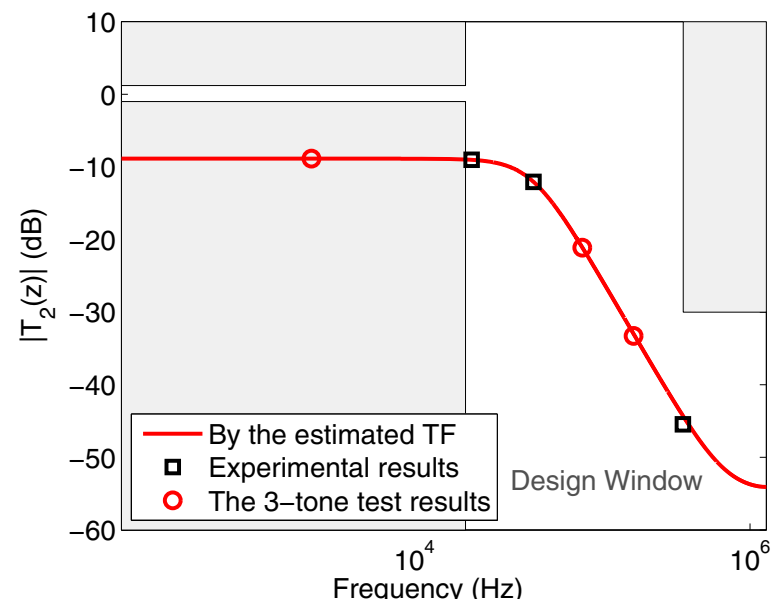

Figure 8. Experimental results when the switch $S G o$ is open.

[7] S. Mir, A. Rueda, T. Olbrichl, E. Peraliast, and J. L. Huertas, "SWITTEST: automatic switch-level fault simulation and test evaluation of switched-capacitor systems," in Proc. IEEE/ACM Design Automation Conf. (DAC), 1997, pp. 281-286.

[8] L. Carro, E. Cota, M. Lubaszewski, Y. Bertrand, F. Azais, and M. Renovell, "TI-BIST: a temperature independent analog BIST for switchedcapacitor filters," in IEEE Asian Test Symp. (ATS), 2000, pp. 78-83.

[9] A. Petraglia, J. Canive, and M. Petraglia, "Efficient parametric fault detection in switched-capacitor filters," IEEE Design \& Test of Computers, vol. 23 , no. 1 , pp. 58-66, Dec. 2006.

[10] H.-C. Hong, "A Static Linear Behavior Analog Fault Model for Switched-Capacitor Circuits," IEEE Trans. Computer-Aided Design of Integrated Circuits and Systems, vol. 31, no. 4, pp. 597-609, 2012.

[11] N. Nagi, A. Chatterjee, A. Balivada, and J. A. Abraham, "Fault-based automatic test generator for linear analog circuits," in Proc. IEEE/ACM Int. Conf. Computer-Aided Design (ICCAD), 1993, pp. 88-91.

[12] Z. You, E. Sanzhez-Sinencio, and J. de Gyvez, "Test generation for linear time-invariant analog circuits," IEEE Trans. Instrumentation and Measurement, vol. 44, no. 1, pp. 28-35, Jan. 1995.

[13] R. Voorakaranam and et al., "Hierarchical specification-driven analog fault modelling for efficient simulation and diagnosis," in Proc. Int. Test Conf. (ITC), 1997, pp. 903-912.

[14] S. F. Hung, L. Y. Lin, and H.-C. Hong, "A study on the Design of a Testable Fleisher-Laker Switched-Capacitor Biquad," in Proc. Int. Mixed-Signals, Sensors, and System Testing Workshop (IMS3TW), 2012, pp. 119-122.

[15] B. Razavi, Design of analog CMOS integrated circuits. New York, NY 10020: McGraw-Hill, 2001. 